\title{
EFFECT OF COMPRESSION ON THE EFFECTIVE THERMAL CONDUCTIVITY AND THERMAL CONTACT RESISTANCE IN PEM FUEL CELL GAS DIFFUSION LAYERS
}

\author{
Ehsan Sadeghi \\ Department of Mechanical Engineering \\ University of Victoria \\ ehsans@uvic.ca
}

\author{
Ned Djilali \\ Department of Mechanical Engineering \\ University of Victoria \\ ndjilali@uvic.ca
}

\author{
Majid Bahrami \\ Mechatronic Systems Engineering \\ Simon Fraser University \\ mbahrami@sfu.ca
}

\section{ABSTRACT}

Heat transfer through the gas diffusion layer (GDL) of a PEM fuel cell is a key process in the design and operation a PEM fuel cell. The analysis of this process requires determination of the effective thermal conductivity as well as the thermal contact resistance between the GDL and adjacent surfaces/layers.

In the present study, a guarded-hot-plate apparatus has been designed and built to measure the effective thermal conductivity and thermal contact resistance in GDLs under vacuum and atmospheric pressure. Toray carbon papers with the porosity of $78 \%$ and different thicknesses are used in the experiments under a wide range of compressive loads. Moreover, novel analytical models are developed for the effective thermal conductivity and thermal contact resistance and compared against the present experimental data. Results show good agreements between the experimental data and the analytical models. It is observed that the thermal contact resistance is the dominant component of the total thermal resistance and neglecting this phenomenon may result in enormous errors.

\section{NOMENCLATURE}

$a=$ Major semi-axis of contact area, $m$

$A \quad=\quad$ Cross-sectional area, $m^{2}$

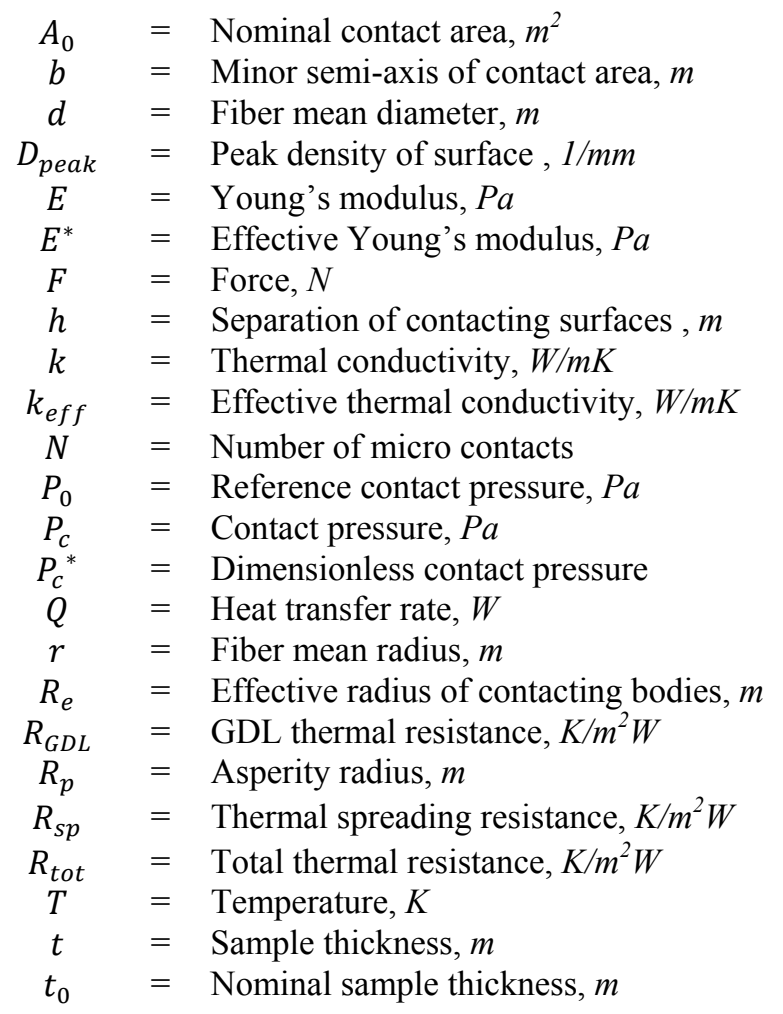




$$
\begin{aligned}
T C R & =\text { Thermal contact resistance, } K / m^{2} W \\
Z & =\text { Height, } m
\end{aligned}
$$

\section{Greek symbols}

$\begin{aligned} \alpha & =\text { Aspect ratio of the contact area, } b / a \\ \varepsilon & =\text { Porosity } \\ \varepsilon_{0} & =\text { Nominal porosity } \\ \epsilon & =\text { Strain, } \Delta t / t_{0} \\ \sigma & =\text { Root mean square of the surface roughness } \\ v & =\text { Poisson's ratio } \\ \lambda & =\text { Onset of elastic deformation, } m \\ \delta & =\text { Deviation in parameters } \\ \delta_{e} & =\text { Elastic deformation, } m \\ \delta_{s} & =\text { Thickness reduction caused by fiber slippage, } \\ & \\ \delta_{\text {tot }} & =\text { Total thickness reduction, } m \\ \kappa(\cdot) & =\text { Elliptic integral of the first kind } \\ \psi(\cdot) & =\text { Constriction parameter } \\ \phi(\cdot) & =\text { Normal distribution }\end{aligned}$

$\begin{array}{cll}\text { Subscripts } & \\ 1 & = & \text { Sample 1 } \\ 2 & = & \text { Sample 2 } \\ c & = & \text { Carbon fiber } \\ f l & = & \text { Fluxmeter } \\ \text { low } & = & \text { Lower contact surface } \\ \text { up } & = & \text { Upper contact surface }\end{array}$

\section{INTRODUCTION}

The electrochemical reaction in proton exchange membrane fuel cell (PEMFC) generates a large amount of heat that results in temperature variation in fuel cell components [1-4]. The heat produced has to be extracted from the cell to maintain the optimum working conditions, developing efficient and reliable cooling strategies for PEMFCs are crucial for efficient and smooth operation of PEMFCs. Accurate knowledge of temperature distribution and associated heat transfer are also required in predicting other transport phenomena such as water and species transport, reaction kinetics, transport properties, rate of phase change, relative humidity, and membrane water content. In addition, thermal-related phenomena in the gas diffusion layer (GDL) and the catalyst layer directly cause hygro-thermal stresses and material degradation which in turn leads to efficiency loss and ultimately mechanical failure $[5,6]$. Any successful fuel cell thermal analysis requires: i) effective thermal conductivity of the gas diffusion layer (GDL) as a function of the micro structural geometry of the GDL and the operating conditions, e.g. compressive load and temperature and ii) thermal contact resistance (TCR), an interfacial phenomenon which occurs due to imperfect contact at GDLbipolar plate and GDL-catalyst layer interfaces. The TCR gives rise to a significant resistance which acts against heat transfer through GDL. Generally, all surfaces have roughness and outof-flatness in microscale, thus the actual contact area is a fraction of the nominal contact area [12]. In GDLs with high porosity, this is even worse; it is expected that the actual contact area to be less than $1 \%$ of the nominal cross-sectional area. In addition, the complexity and anisotropy of GDL microstructure make it intricate to define accurate values for TCR and the effective thermal conductivity.

Large differences in thermal conductivity of solid and fluid phases as well as high porosity of GDL micro structure make it necessary to define an effective thermal conductivity. This transport property plays an important role in fuel cell performance analysis [7] and is required in computational models [8]. There are few studies in the literature which have focused on the analytical modeling of GDL thermal conductivity. Ramousse et al. [7] investigated the effective thermal conductivity of non-woven carbon felt GDLs and estimated the conductivity bounds using a model connecting the two phases (solid and gas) in series or parallel. They used Danes and Bardon [9] correlation to estimate the effective thermal conductivity of the solid phase. The model as well as the experimental measurements yielded conductivity values that are lower than most values reported in the literature. Using the unit cell concept, the present authors recently presented a compact analytical model to determine the effective thermal conductivity of GDLs [10]. A microstructure of uniformly sized, equally spaced cylindrical fibers immersed in stagnant air was assumed, and the Hertzian theory was used to calculate the contact area between the touching fibers, considering a range of fiber angles. The analysis was performed by constructing a thermal resistance network that takes into account the thermal paths through solid fibers and air.

The complexity of analytical modeling of GDL microstructure leads researchers toward numerical and experimental works. Khandelwal and Mench [11] measured the through-plane thermal conductivity of GDLs. They examined two different types of commercial GDLs with a variety of thickness and porosity. They studied the effect of temperature and polytetrafluoro ethylene (PTFE) content on the effective thermal conductivity, and obtained values in close agreement with the manufacturer data sheet.

Nitta et al. [12] measured the GDL thickness under compressive loads. They used a guarded-hot-plate apparatus to measure the effective thermal conductivity and thermal contact resistance of GDL (SGL SIGRACET®10 BA) at different pressures. They reported that the GDL thermal conductivity is independent of the compression. To deduce the thermal conductivity and TCR data, they assumed that the contact resistance between GDL layers is negligible. To eliminate this thermal contact resistance, conductive silver particles were sputtered onto the GDL surfaces.

Burheim et al. [13] measured the effective thermal conductivity and TCR of uncoated SolviCore gas diffusion layer at various compaction pressures using similar apparatus as Nitta et al.'s [12]. They showed that the effective thermal conductivity increases but TCR decreases with an increase in the compressive load.

The available studies on thermal contact resistance of GDLs in the literature are limited to experimental measurements and there is a lack for analytical investigations in this field. However, several pertinent analytical and experimental approaches have been reported on electrical contact resistance [14-17]. These studies have employed fractal based models [14] or the Hertzian elastic theory [15-17] to find the contact area 
between the asperities of GDL and bipolar plate/catalyst layer surfaces and have the potential of being extended to thermal analysis.

Our literature review indicates that in the majority of the previous related-studies on the heat transfer in GDL, the TCR was bundled up with the effective thermal conductivity and only one value is reported. It should be noted that the TCR is an "interfacial phenomenon" and is a function of mechanical load and surface characteristics of both mating surfaces where as thermal conductivity is a transport property of a "continuous medium". Therefore, thermal conductivity and TCR should be treated separately. The effect of compressive load on thermal conductivity and TCR has not been thoroughly investigated. Our experimental results show that the TCR is the dominant component of the total thermal resistance of a layer of GDL sandwiched between two solid bodies. In the present study, a comprehensive experimental and analytical study is conducted to determine through-plane thermal conductivity of GDLs as well as the thermal contact resistance at the interface of GDL and a solid surface as a function of the compressive load.

A custom-made test bed was designed and built that enables the measurements of thermal conductivity and TCR of porous media under vacuum and ambient pressure conditions. The test bed is equipped with a loading mechanism that allows the application of various compressive loads on the samples. Toray carbon papers with the porosity of $78 \%$ and different thicknesses are used in the experiments. The effect of ambient and compression is investigates, and includes measurement of the GDL thickness variation using a tensile-compression apparatus. The effective thermal conductivity and TCR are deduced from the total thermal resistance measurements by performing a series of experiments with GDL samples of various thickness and similar microstructure. The effect of operating temperature $\left(35-70^{\circ} \mathrm{C}\right)$ on both thermal conductivity and TCR is also investigated. Furthermore, analytical models are developed to evaluate through-plane thermal conductivity of GDLs as well as the thermal contact resistance at the interface of GDL and a solid surface as a function of the compressive load. These models are compared against experimental data obtained in this study.

\section{EXPERIMENTAL STUDY}

\subsection{Thickness Measurement}

The thickness variation of Toray carbon papers TGP-H-060 and TGP-H-120 under different compressive loads was measured using a tensile compression apparatus. A Mitutoyo digital indicator with the resolution of $0.001 \mathrm{~mm}$ was used to measure the thickness variation under the load. The GDL samples are cut in a circular shape with the diameter of $25 \mathrm{~mm}$ and then compressed by a steel rod using a pneumatic actuator. Various compression forces were applied on the GDL using the apparatus. The load was increased at 15-20 min intervals to ensure a mechanical equilibrium and steady-state condition. Measurements were repeated five times for each sample and the averaged values for thickness and porosity are reported in Fig. 1.

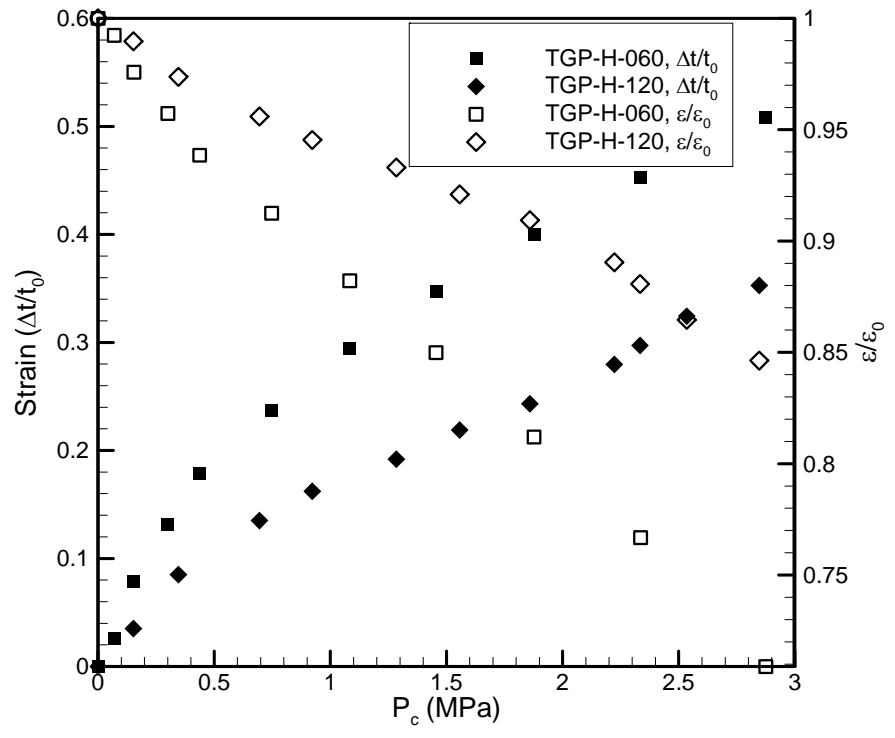

\section{FIGURE 1. THICKNESS AND POROSITY VARIATION OF TORAY CARBON PAPERS UNDER COMPRESSION.}

\subsection{Thermal Measurement}

A schematic of the test column in the test chamber is shown in Fig. 2. The test chamber consists of a stainless steel base plate and a bell jar enclosing the test column. The test column consists of, from top to bottom: the loading mechanism, the steel ball, the heater block, the upper heat fluxmeter, the sample, the lower fluxmeter, the heat sink (cold plate), the load cell, and the poly methyl methacrylate (PMMA) layer. The heater block consists of circular flat copper in which cylindrical pencil-type electrical heaters are installed. The power of the heaters can be adjusted manually. In the present study, a $30 \mathrm{~W}$ Omega heater is used.

Cooling is accomplished using a closed loop water-glycol bath in which the coolant temperature can be set. A $1000 \mathrm{lbs}$ load cell is used to measure the applied load to the joint. The load is applied over a load button placed at the center of the load cell.

The fluxmeters were made from a standard electrolyte iron material. To measure temperatures six T-type thermocouples were attached to each fluxmeter at specific locations shown in Fig. 2. The thermocouples were located $5 \mathrm{~mm}$ apart with the first one $10 \mathrm{~mm}$ from the contact surface. The thermal conductivity of the iron fluxmeter was known and used to measure the heat flow rate transferred through the contact region.

Toray carbon papers TGP-H-120 and TGP-H-60 with the porosity of $78 \%$ were used. These samples have $5 \%$ wet proofing and their thicknesses are $0.37 \mathrm{~mm}$ and $0.19 \mathrm{~mm}$, respectively. The samples were cut in circles with $25 \mathrm{~mm}$ diameter and sandwiched between the fluxmeters.

Experiments are conducted under vacuum and ambient conditions. A vacuum level of $10^{-5} \mathrm{mbar}$ is achieved under the test chamber using the vacuum machine. To minimize heat transfer to the surrounding, the test column including the fluxmeters and samples were insulated using glass wool 
insulation layers. Temperatures and pressure are recorded at various compressive loads when steady-state condition is achieved; to reach thermal equilibrium all the experiment's parameters are kept constant and carefully monitored for approximately 4-5 hours for each data point.

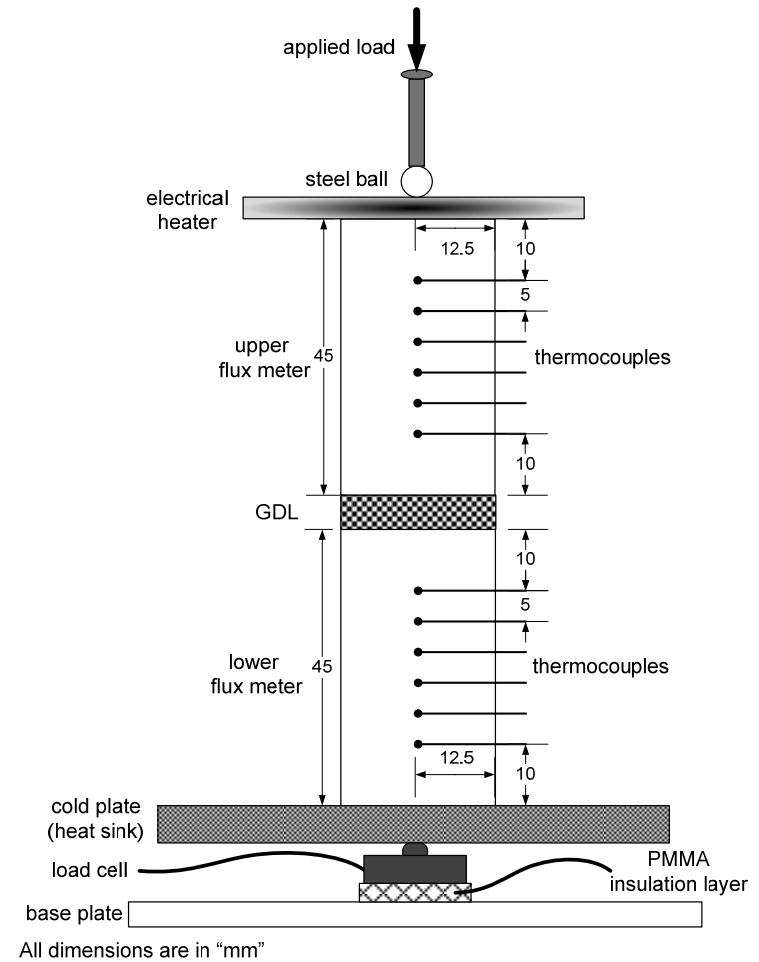

FIGURE 2. SCHEMATIC VIEW OF THE TEST COLUMN.

The temperature gradient between the hot and the cold plates causes a 1-D heat conduction from top to the bottom in the test column. It is also assumed that the natural convection within the GDL sample is negligible for atmospheric pressure tests because of the heat flow direction and also small Grashof number; for a typical GDL, the Grashof number is in order of $10^{-6}$ and is significantly lower than 2500 , the limit for natural convection [18].

The radiation heat transfer between the fibers is also negligible because of small temperature difference between the fibers and also the temperature levels in the samples during the tests, i.e. less than $100^{\circ} \mathrm{C}$. Thus, the heat transfer is only due to conduction through the fibers and air (atmospheric tests) and can be determined using Fourier's equation.

$$
Q=-k A \frac{d T}{d z}
$$

where, $d T / d z$ is the temperature gradient along the test column and $k$ is the thermal conductivity of the fluxmeters. Temperatures at top and bottom contact surfaces can be extrapolated through the measured heat flux. The total thermal resistance of the sample, $R_{t o t}$, includes the sample thermal resistance and the thermal contact resistance (at the top and bottom surfaces) and can be expressed as:

$$
R_{t o t}=R_{G D L}+T C R=\frac{\Delta T_{u l}}{Q}
$$

where, $\Delta T_{u l}$ is the temperature difference between the upper and the lower contact surfaces. $R_{G D L}$ and TCR are the GDL resistance and the total contact resistance, respectively. There are two interface between the GDL and fluxmeter; it is assumed that the contact resistance at the top and bottom of GDLs are equal; $T C R_{\text {up }}=T C R_{\text {low }}=T C R / 2$.

To find both thermal conductivity and TCR, two experiments were performed with samples of different thicknesses; samples with identical microstructural parameters were used. Under the same pressure, TCR for both samples is assumed to be equal. Applying Eq. (2) to both of the measurements and subtracting them, one can find the effective thermal conductivity:

$$
\begin{gathered}
k_{e f f}=\frac{t_{1}}{R_{G D L 1} A}=\frac{t_{2}}{R_{G D L 2} A} \\
k_{e f f}=\frac{t_{1}-t_{2}}{\left(R_{t o t 1}-R_{\text {tot } 2}\right) A}
\end{gathered}
$$

where, $t_{1}$ and $t_{2}$ are thicknesses of sample 1 and 2 at the specific applied pressure and $A$ is the cross-section of samples. Equation (4) can be used to find the effective thermal conductivity; the TCR can then be calculated by Eq. (2).

\subsection{Uncertainty Analysis}

Considering relationships for evaluating the effective thermal conductivity and the thermal contact resistance, i.e. Eqs.(4), (2), the parameters involved in the analysis can be expressed as:

$$
R_{\text {tot }}=f\left(Q, \Delta T, t, A, P_{c}\right)
$$

The main uncertainty in this experiment is due to errors in estimation of heat flux passing the sample which causes the maximum error of $4.3 \%$. The maximum uncertainties for thermocouples and the data acquisition readings are $\pm 1^{\circ} \mathrm{C}$ which introduces the maximum error of $1.3 \%$ between the interfaces of the sample and fluxmeters. Other uncertainties including the uncertainty of the load cell and the uncertainty in measuring the thickness under compression and the crosssectional area are listed in Table 1. The maximum uncertainties for thermal resistance measurements can be calculated from [19]:

$$
\begin{aligned}
& \frac{\delta R_{t o t}}{R_{t o t}} \\
& =\sqrt{\left(\frac{\delta Q}{Q}\right)^{2}+\left(\frac{\delta \Delta T}{\Delta T}\right)^{2}+\left(\frac{\delta t}{t}\right)^{2}+\left(\frac{\delta A}{A}\right)^{2}+\left(\frac{\delta P_{c}}{P_{c}}\right)^{2}}
\end{aligned}
$$

For the present study, the uncertainty is estimated to be $\pm 6 \%$.

Table 1. UNCERTAINTY OF INVOLVED PARAMETERS IN THE ANALYSIS.

\begin{tabular}{|c|c|c|c|c|}
\hline $\boldsymbol{\delta Q} / \mathbf{Q}$ & $\boldsymbol{\delta} \Delta \mathbf{T} / \Delta \mathbf{T}$ & $\boldsymbol{\delta t} / \mathbf{t}$ & $\boldsymbol{\delta A} / \mathbf{A}$ & $\boldsymbol{\delta}_{\mathbf{c}} / \mathbf{P}_{\mathbf{c}}$ \\
\hline $4.3 \%$ & $1.3 \%$ & $2.7 \%$ & $1.6 \%$ & $2.5 \%$ \\
\hline
\end{tabular}




\section{ANALYTICAL MODELLING}

\subsection{Through-Plane Thermal Conductivity}

To determine the through-plane effective thermal conductivity of fibrous GDL, a unit cell approach is employed [10]. The goal of this approach is to model the random and anisotropic structure of GDL with a relatively simple geometry which can predict the effective thermal conductivity accurately. The proposed geometrical model is shown in Fig. 4 and consists of uniformly sized equally spaced cylindrical fibers immersed in stagnant air. The fibers angle, $\theta$, can be varied in this model. Although the fibers are randomly oriented in practice, the averaged effect of this randomness on the transport properties of a sample is well represented by $\mathrm{n}$ unit cell with an orthogonal arrangement as shown in Sadeghi et al. [10] comparison of model predictions and experiments; this is corroborated by the recent results of Van Doormaal et al. [21]. Thus, in the present study, the orthogonal and square arrangement of fibers is considered. The microstructure of carbon papers is deformed non-linearly with the compressive load as shown in Fig. 1. This non-linear deformation is a complex combination of elastic and plastic deformations and slippage and breakage of fibers, binders, and PTFE. We modeled this deformation as a combination of elastic deformation and slipping of fibers. A schematic of the deformation of the unit cell under the load is shown in Fig. 4. The total thickness reduction is the summation of elastic deformation and thickness variation as a result of fiber slippage.

$$
\delta_{t o t}=\delta_{s}+\delta_{e}=\epsilon . d
$$

where, $d$ is the mean diameter of fibers and $\delta_{e}$ and $\delta_{s}$ are the thickness reductions as a result of elastic deformation and fiber slippage, respectively. The deformation of the carbon paper under the load shown in Fig. 3 is correlated by:

$$
\begin{aligned}
& \epsilon=\frac{\Delta t}{t_{0}} \\
& = \begin{cases}0.274\left[1-\exp \left(-0.988 P_{c}^{*}\right)\right] & : \mathrm{TGP}-\mathrm{H}-120 \\
0.449\left[1-\exp \left(-1.063 P_{c}^{*}\right)\right] & : \mathrm{TGP}-\mathrm{H}-060\end{cases}
\end{aligned}
$$

where, $P_{c}^{*}$ is the contact pressure in MPa non-dimensionalized with respect to the reference pressure $P_{0}=1 \mathrm{MPa}$.

To find the contact area between fibers, the Hertzian contact theory [20] is applied, see [10] for more details. Based on this theory, when a cylindrical fiber contacts another cylindrical fiber eccentrically, as in this study, the contact spot is close to an ellipse and the relation between radii of contact area $a, b$ and the load F can be expressed approximately in the terms of deformations as,

$$
F=\frac{4}{3} E^{*} R_{e}^{1 / 2} \delta_{e}^{3 / 2}
$$

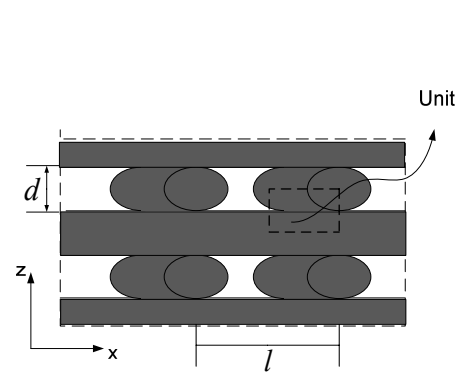

(a)

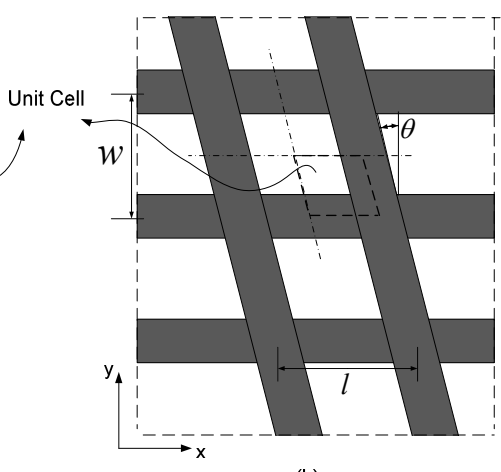

(b)
FIGURE 3. GEOMETRICAL MODEL OF GDL: (a) FRONT VIEW; (b) TOP VIEW [10].

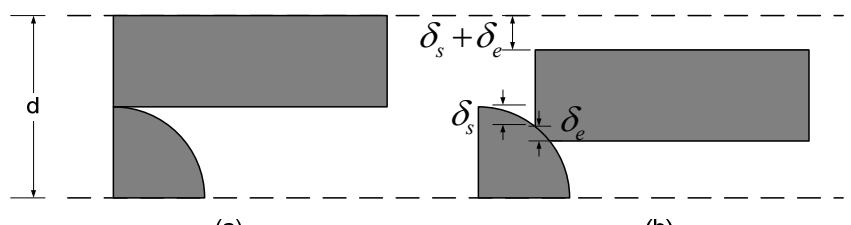

(a)

(b)

FIGURE 4. CONTACTING FIBERS IN THE UNIT CELL: (a) BEFORE COMPRESSION; (b) AFTER COMPRESSION.

where, $R_{e}$ is the equivalent radius of the principal radii of curvature of two contacting bodies which is equal to the average fiber radius for the present study. $E^{*}$ is the effective Young's modulus which can be defined as a function of Young's modulus and Possion's ratio of two contact bodies.

$$
E^{*}=\left(\frac{1-v_{1}^{2}}{E_{1}}+\frac{1-v_{2}^{2}}{E_{2}}\right)^{-1}
$$

The major and minor radii of the contact area can be written as:

$$
\begin{gathered}
a=\sqrt{r^{2}-\left(r-\delta_{e}\right)^{2}} \\
b=\sqrt{r^{2}-\left(r-\delta_{e}-\lambda\right)^{2}}-\sqrt{r^{2}-(r-\lambda)^{2}}
\end{gathered}
$$

where, $\lambda$ is the onset of elastic deformation, the thickness variation before the start of the elastic deformation. Through a comparison with experimental data, $\lambda$ is found to be $\delta_{s} / 60$ and $\delta_{s} / 15$ for TGP-H-060 and TGP-H-120, respectively. Comparison of different thermal resistances against the heat transfer in the unit cell indicates that the constriction/spreading resistance $R_{s p}$ is the controlling resistance [10]. Thus to develop a compact model, the contributions of other resistances can be neglected. When heat flows in/out of a body through a small area, the heat flux lines are correspondingly constricted/spread apart and the resulting thermal resistance is referred to as constriction/spreading resistance. The spreading resistance can be approximated by the solution of an elliptical heat source on a circular flux tube given by [22]: 


$$
R_{s p}=\frac{1.6974 \psi(\alpha)}{\pi^{2} k_{s} b} \kappa\left(1-\frac{a^{2}}{b^{2}}\right)
$$

where, $\psi(\alpha)$ is the constriction parameter which can be expressed as [23]:

$$
\psi(\alpha)=(1-\alpha)^{1.5}
$$

where, $\alpha$ is the ratio of the contact size to the fiber radius $\alpha=\sqrt{a b} / r . \kappa(\cdot)$ is the complete elliptic integral of the first kind defined as:

$$
\kappa\left(1-\frac{a^{2}}{b^{2}}\right)=\int_{0}^{\pi / 2} \frac{d t}{\sqrt{1-\left(1-\frac{a^{2}}{b^{2}}\right) \sin ^{2} t}}
$$

The effective thermal conductivity of GDL can be found through:

$$
k_{e f f}=\frac{d(1-\epsilon)}{2 R_{s p} A}
$$

where, $A$ is the cross-sectional area of the unit cell, $A=w^{2} / 4$.

\subsection{Thermal Contact Resistance}

All surfaces are inherently rough and the real contact area occurs microscopically between asperities of two contacting bodies. Therefore, the topologies of both contacting surface are important in understanding their interfacial behavior [15]. To verify our experimental data for TCR, an analytical model is developed using the Greenwood and Williamson statistical model [24]. This model is based on the Hertz solution for individual elastic contacts and assumes that only asperities originally higher than the separation of the surfaces are in contact.

Surface roughness of fluxmeters and carbon papers are measured using a Mitutoyo profilometer. The results show that the average roughnesses for the fluxmeters are less than $1 \mu \mathrm{m}$, therefore, their surfaces can be assumed smooth. For carbon papers with high porosity and random fiber distribution in the surface, it is complicated to define roughness parameters. In this study, we assumed that the carbon paper surface acts as a rough solid surface and we found their roughness parameters through profilometry. The measured parameters, average data for TGP-H-120 and TGP-H-060, are $\sigma=8.96 \mu \mathrm{m}$ and $D_{\text {peak }}=12.6 \mathrm{~mm}^{-1}$. The asperity radius is assumed to be equal as the average fiber radius, $R_{p}=r=4.25 \mu \mathrm{m}$. The total contact force can be found by [24]:

$$
F=P_{c} A_{0}=N \int_{h}^{\infty} \frac{4}{3} E^{*} R_{e}^{\frac{1}{2}}(z-h)^{\frac{3}{2}} \phi(z) d z
$$

where, $N$ is the total number of contact points and $h$ is the separation of the surfaces. $P_{c}$ and $A_{0}$ are the contact pressure and the nominal contact area, respectively. $\phi(z)$ is the normal distribution of the surface height which can be described as:

$$
\phi(z)=\frac{1}{\sigma \sqrt{2 \pi}} \exp \left(-\frac{z^{2}}{2 \sigma^{2}}\right)
$$

where, $\sigma$ is the root mean square of the surface roughness. Since the deformation of GDL under compression is significant, a portion of the force $F$ is used for the thickness reduction. To find the actual contact area, the thickness reduction of the unit cell close to the contact surface should be subtracted from the total deformation. The total contact resistance can be expressed as:

$$
T C R=\left(\frac{2 N k_{f l} k_{c} \int_{h+\epsilon d}^{\infty} \sqrt{(z-h-\epsilon d) R_{p}} \phi(z) d z}{k_{f l}+k_{c}}\right)^{-1}
$$

where, $k_{f l}$ and $k_{c}$ are thermal conductivities of the fluxmeter and the carbon fiber, respectively. The input data required for the TCR model are reported in Table 2.

Table 2. MECHANICAL AND THERMAL PROPERTIES OF CARBON FIBER AND FLUXMETER.

\begin{tabular}{|c|c|c|c|}
\hline $\mathbf{E}_{\mathbf{c}}(\mathbf{G P a})$ & $\mathbf{E}_{\mathbf{f l}}(\mathbf{G P a})$ & $\mathbf{k}_{\mathbf{c}}\left(\mathbf{W} \mathbf{m}^{-\mathbf{1}} \mathbf{K}^{-\mathbf{1}}\right)$ & $\mathbf{k}_{\mathbf{f l}}\left(\mathbf{W} \mathbf{m}^{-\mathbf{1}} \mathbf{K}^{-\mathbf{1}}\right)$ \\
\hline 3.2 & 210 & 120 & 66 \\
\hline
\end{tabular}

\section{RESULTS AND DISCUSSION}

The measurements have been taken at different compressive loads in a vacuum and in atmospheric air pressure to study the effects of the compressive load and heat conduction in air on TCR and the effective thermal conductivity.

The effective thermal conductivity values are compared with the analytical model, Eq. (16), in Fig. 5 for vacuum and atmospheric pressure. The effective conductivity increases with an increase in the compressive load due to reduced porosity and larger contact areas between the touching fibers. The reported value for the thermal conductivity by the manufacturer is 1.7 $\mathrm{W} / \mathrm{mK}$ which differs $4.4 \%$ from our result at a relatively low pressure of $0.478 \mathrm{MPa}$. A small difference is observed between thermal conductivity values in vacuum and atmospheric pressure which shows that the air trapped in the pores of the medium provides an additional path for the heat conduction. Also, a comparison between the model and experimental data shows a good agreement for both vacuum and atmospheric pressure over a wide range of the compressive load.

Figure 6 shows thermal contact resistance of both Toray carbon papers at different contact loads under vacuum and the atmospheric pressure. Also, the present analytical model for TCR at vacuum condition, Eq. (19) is compared to the experimental data in Fig. 6. As seen, the model provides a good estimation for TCR and all data are within $15 \%$ of the analytical model. Since air fills the gap between the contact surfaces and provides another path for heat conduction across the contact interface, the thermal contact resistance and consequently total thermal resistance decreases. This reduction 
is less for high contact pressure because of the higher contact area providing the preferable thermal path. In both air pressure conditions, TCR decreases with an increase in the compressive load due to larger (or increased) contact area. Also, increasing the contact pressure produces fiber breakage and irreversible deformations [25] which create hysteresis effects under cyclic loads.

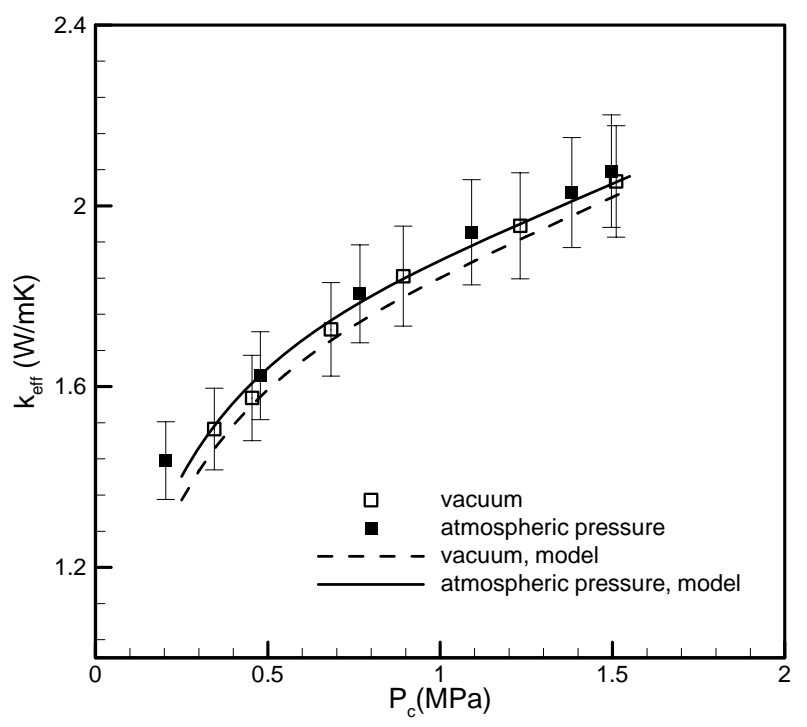

FIGURE 5. EFFECTIVE THERMAL CONDUCTIVITY OF THE TORAY CARBON PAPERS AT VACUUM AND ATMOSPHERIC PRESSURES: EXPERIMENTAL AND ANAYTICAL RESULTS.

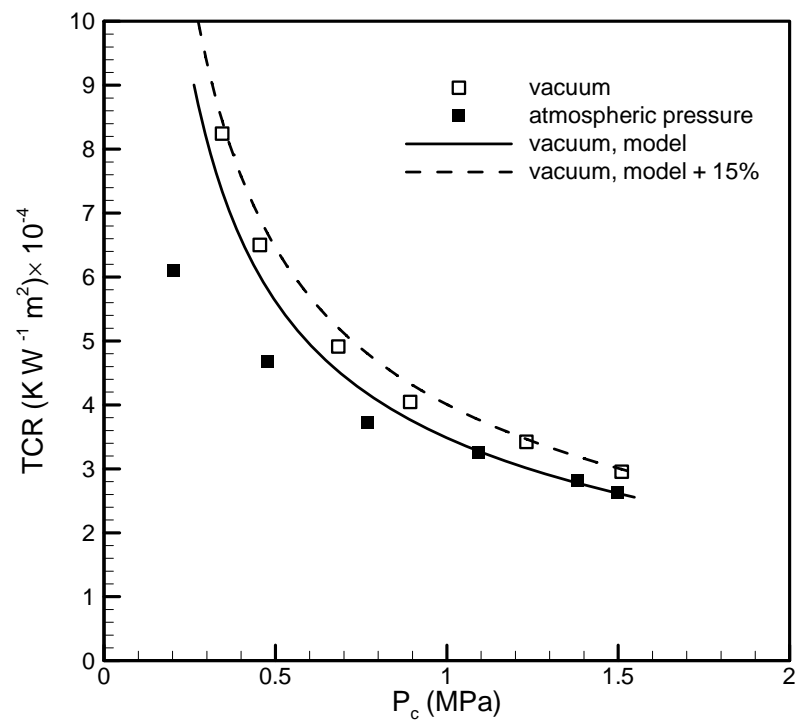

FIGURE 6. THERMAL CONTACT RESISTANCE: ANALYTICAL MODEL AND EXPERIMENTAL DATA.

Figure 7 shows the TCR to total resistance ratio as a function of compression, and we observe that:
- TCR is clearly the dominant resistance, contributing between 65 and $90 \%$ of the total resistance.

- As expected, the relative contribution of TCR is more important for thinner materials; the average TCR ratio for TGP-H-120 and TGP-H-060 at atmospheric pressure is $68 \%$ and $82 \%$, respectively.

Both thermal conductivity and TCR decrease with increasing compression; however, as shown in Fig. 7, the TCR to total resistance ratio remains approximately constant.

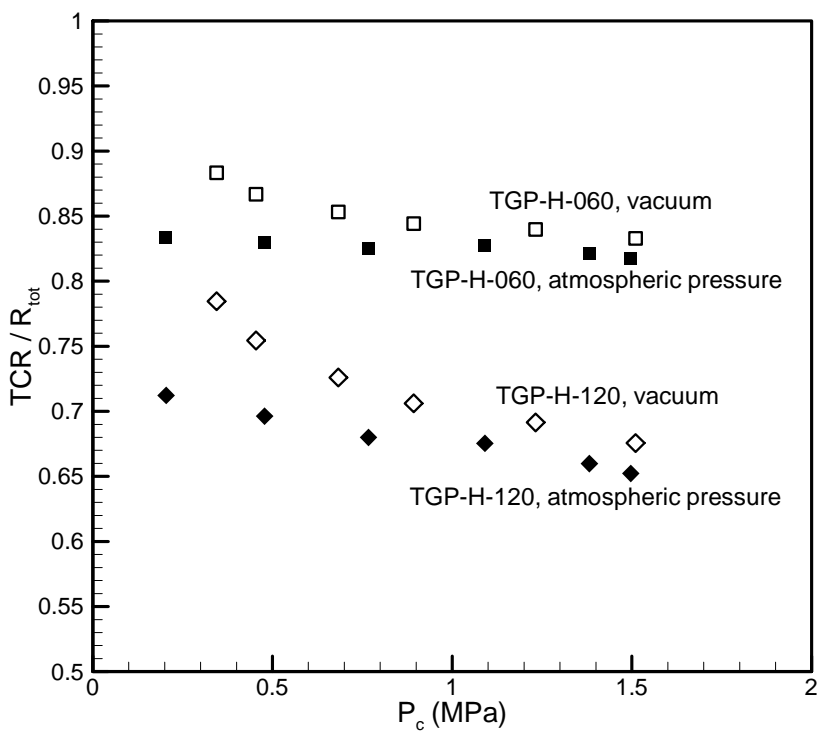

\section{FIGURE 7. THERMAL CONTACT RESISTANCE TO TOTAL RESISTANCE RATIO AT DIFFERENT COMPRESSIVE LOADS.}

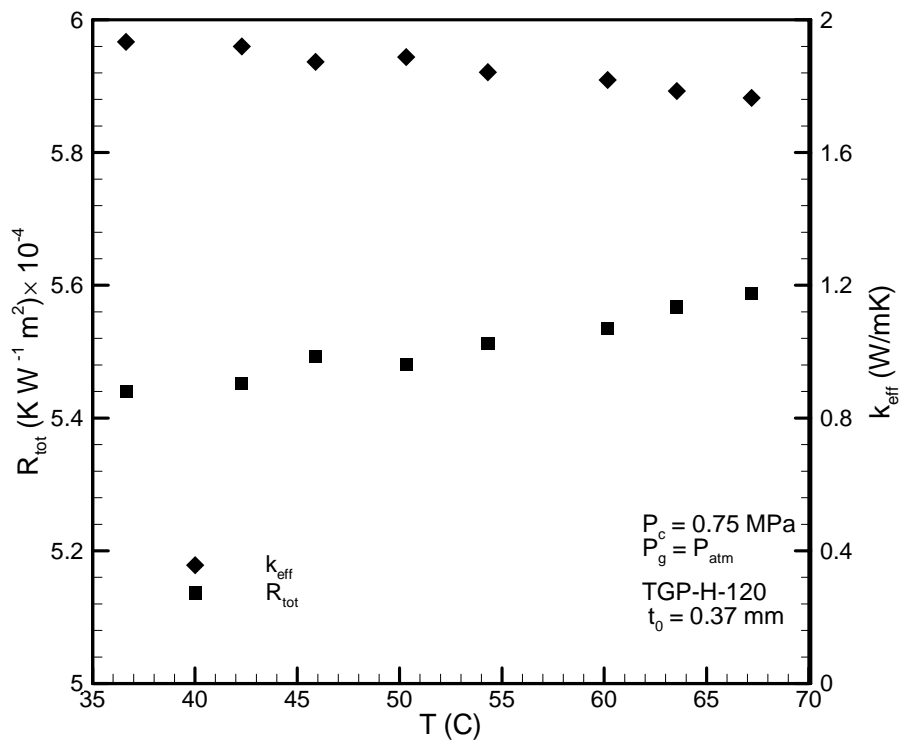

FIGURE 8. EFFECT OF OPERATION TEMPERATURE ON THE THERMAL CONTACT RESISTANCE AND EFFECTIVE THERMAL CONDUCTIVITY. 
The variations of the total thermal resistance and of the effective thermal conductivity with temperature are shown in Fig. 8 for TGP-H-120 sample subjected to a constant contact pressure of $0.75 \mathrm{MPa}$. The effective thermal conductivity decreases slightly with increasing temperature, while the total resistance remains approximately constant. Considering that the TCR is the controlling component of the total resistance, we can conclude that the TCR does not depend on temperature, at least in the range of temperatures considered here.

The reduction in thermal conductivity can attributed to the presence of carbonized thermo setting resins used as a binder in GDLs [26]. The thermal conductivity of these thermo-setting polymers decreases with increasing temperature [27], and this would result in a reduction in the effective thermal conductivity of the medium.

\section{SUMMARY AND CONCLUSIONS}

A test bed was designed and built and analytic models were developed to measure and predict thermal conductivity and thermal contact resistance of GDLs under various compressive loads. The model predictions are in good agreement with experimental data over a wide range of compressive loads from 0.2 to $1.5 \mathrm{MPa}$. Parametric studies have been performed to investigate the trends and effects of compression, conduction in air, and operating temperature. The highlights of the results are:

- The effective thermal conductivity increases with the compressive load and decreases with an increase with operating temperature, but that it is relatively insensitive to ambient air pressure.

- Thermal contact resistance is the controlling component of the total thermal resistance.

- the ratio of thermal contact to bulk GDL resistance remains approximately constant, e.g. (4.6/1) for TGP$\mathrm{H}-060$ at atmospheric pressure over a range of conditions.

This work has helped clarify the impact of several operational parameters on the thermal properties of GDLs and provided new insights on the importance of a key interfacial phenomenon.

\section{ACKNOWLEDGMENTS}

The authors are grateful for the financial support of the Natural Sciences and Engineering Research Council (NSERC) of Canada, and the Canada Research Chairs Program.

\section{REFERENCES}

[1] Djilali, N. and Lu, D., 2002, "Influence of heat transfer on gas and water transport in fuel cells", Int. J. Therm. Sci., Vol. 41, pp. 29-40.

[2] Berning, T. and Djilali, N., 2003, "A 3D, Multiphase, Multicomponent model of the cathode and anode of a PEM fuel cell”, J. Electrochem. Soc., Vol. 150, no. 12, pp. A1589-A1598. [3] Hea, S., Mench, M.M., and Tadigadapa, S., 2006, “Thin film temperature sensor for real-time measurement of electrolyte temperature in a polymer electrolyte fuel cell",
Sensors and Actuators A: Physical, Vol. 125, no. 2, pp. 170177.

[4] Vie, P.J.S. and Kjelstrup, S., 2004, "Thermal conductivities from emperature profiles in the polymer electrolyte fuel cell", Electrochim. Acta, Vol. 49, pp. 1069-1077.

[5] Kusoglu, A., Tang, Y., Santare, M.H., Karlsson, A.M., Cleghorn, S., and Johnson, W.B., 2009, "Stress-Strain Behavior of Perfluorosulfonic Acid Membranes at Various Temperatures and Humidities: Experiments and Phenomenological Modeling", J. Fuel Cell Sci. Technol., Vol. 6, pp.011012011019.

[6] Kusoglu, A., Karlsson, A.M., Santare, M.H., Cleghorn, S., Johnson, W.B., 2006," Mechanical response of fuel cell membranes subjected to a hygro-thermal cycle", J. Power Sources, Vol. 161, pp. 987-996.

[7] Ramousse, J., Didierjean, S., Lottin, P., and Maillet, D., 2008, "Estimation of the effective thermal conductivity of carbon felts used as PEMFC Gas Diffusion Layers", Int. J. Therm. Sci., Vol. 47, no. 1, pp.1-6.

[8] Hakenjos, A., Muenter, H., Wittstadt, U., and Hebling, C., 2004, "A PEM fuel cell for combined measurement of current and temperature distribution, and flow field flooding", J. Power Sources, Vol. 131, pp. 213-216.

[9] Danes, F. and Bardon, J.P., 1997, "Conductivité thermique des feutres de carbone, isolants à forte anisotropie : modèle de conduction par la phase solide", Revue Générale de Thermique, Vol. 36, pp. 302-311.

[10] Sadeghi, E., Bahrami, M., and Djilali, N., 2008, "Analytic determination of the effective thermal conductivity of PEM fuel cell gas diffusion layers", J. Power Sources, Vol. 179, pp. 200208.

[11] Khandelwal, M. and Mench, M.M., 2006, "Direct measurement of through-plane thermal conductivity and contact resistance in fuel cell materials", J. Power Sources, Vol. 161, pp. 1106-1115.

[12] Nitta, I., Himanen, O., and Mikkola, M., 2008, "Thermal conductivity and contact resistance of compressed gas diffusion layer of PEM fuel cell", Fuel Cells, Vol. 8, no. 2, pp. 111-119.

[13] Burheim, O., Vi, P.J.S., Pharoah, J.G, and Kjelstrup, S., 2010, "Ex situ measurements of through-plane thermal conductivities in a polymer electrolyte fuel cell", J. Power Sources, Vol. 195, pp. 249-256.

[14] Mishra, V., Yang, F., and Pitchumani, R., 2004, "Measurement and Prediction of Electrical Contact Resistance between Gas Diffusion Layers and Bipolar Plate for Applications to PEM Fuel Cells, J. Fuel Cell Sci. Technol., Vol. 1, pp. 2-9.

[15] Zhou, Y., Lin, G., Shih, A.J., and Hu, S.J., 2007, “A Micro-Scale Model for Predicting Contact Resistance between Bipolar Plate and Gas Diffusion Layer in PEM Fuel Cells", $J$. Power Sources, Vol. 163, pp. 777-783.

[16] Wu, Z., Wang, S., Zhang, L., and Hu, S.J., 2009, “An analytical model and parametric study of electrical contact resistance in proton exchange membrane fuel cells", J. Power Sources, Vol. 189, pp. 1066-1073.

[17] Swamy, T., Kumbar, E.C., and Mench, M.M., 2010, "Characterization of Interfacial Structure in PEFCs: Water Storage and Contact Resistance Model", J. Electrochem. Soc., Vol. 157, pp. B77-B85. 
[18] Arpaci, V.S. and Larsen, P.S., 1984, Convection Heat Transfer, Chapt. 4, Prentice-Hall, Englewood Cliffs, NJ.

[19] Taylor, T.R., 1980, An Introduction to Error Analysis -

The Study of Uncertainties in Physical Measurements, University Science Book, Mill Valley.

[20] Johnson, K.L., 1985, Contact Mechanics, Chapt. 4, Cambridge Univ. Press, London, UK.

[21] Van Doormaal, M., 2006, Determination of Permeability in Fibrous Porous Media Using the Lattice Boltzmann Method with Application to PEM Fuel Cells, M. Eng. thesis, Queen's University, Kingston.

[22] Yovanovich, M.M., 1976, "Thermal Constriction Resistance of Contacts on a Half-Space: Integral Formulation", Progress in Astronautics and Aeronautics: Radiative Transfer and Thermal Control, Vol. 49, pp. 397.418.

[23] Cooper, M.G., Mikic, B.B., and Yovanovich, M.M., 1969, "Thermal contact conductance", International Journal of Heat and Mass Transfer, Vol. 12, pp. 279-300.

[24] Greenwood, J.A. and Williamson, B.P., 1966, "Contact of Nominally Flat Surfaces", Proceedings of the Royal Society of London: Series A, Math. Phys. Sci. Vol. 295, pp. 300.319

[25] Bazylak, A., Sinton, D., Liu, Z.-S., and Djilali, N., 2007, "Effect of Compression on Liquid Water Transport and Microstructure of PEMFC Gas Diffusion Layers", J. Power Sources, Vol. 163, pp. 784-792.

[26] Mathias, M.F., Roth, J., Fleming, J., and Lehnert, W., 2003, Handbook of Fuel Cells-Fundamental, Technology and Application, Vol. 3, John Wiley \& Sons, Ltd., pp. 517-537.

[27] Choy, C.L., Wong, Y.W., Yang, G.W., and Kanamoto, T., 1999, "Elastic Modulus and Thermal Conductivity of Ultradrawn Polyethylene", J. Polym. Sci.: Polym. Phys., Vol. 37, pp. 3359-3367. 